\title{
COBERTURAS ASSISTENCIAIS NEGADAS PELOS PLANOS E SEGUROS DE SAÚDE EM AÇÕES JULGADAS PELO TRIBUNAL DE JUSTIÇA DO ESTADO DE SÃO PAULO
}

Coverage assistance denied by plans and health insurance in

lawsuits judged by the Court of Justice of the São Paulo State

Mário Scheffer*

\begin{abstract}
RESUMO
A pesquisa analisou 782 decisões judiciais relacionadas à negação de coberturas assistenciais movidas por clientes de planos e seguros de saúde, julgadas pelo Tribunal de Justiça do Estado de São Paulo em 2009 e 2010. As negativas de tratamento de câncer e de doenças cardiovasculares são os principais motivos das ações judiciais. Dentre os procedimentos médicos mais excluídos destacamse a quimioterapia, radioterapia e cirurgias diversas. Também foram negados exames diagnósticos, medicamentos, órteses e próteses. Em $88 \%$ dos casos o julgamento foi favorável ao usuário, obrigando a empresa de plano de saúde a oferecer a cobertura negada.
\end{abstract}

Palavras-chave: Cobertura de Serviços Privados de Saúde; Poder Judiciário; Sistema de Saúde.

\section{ABSTRACT}

The research analyzed 786 judicial decisions related to the denial of medical coverage assistance moved by customers of plans and health insurance, judged by the Court of Justice of the State of São Paulo in 2009 and 2010. The denial of treatment of cancer and cardiovascular diseases were the main reasons of lawsuits. Among the most excluded medical procedures are chemotherapy,

(*) Doutor em Ciências, Departamento de Medicina Preventiva, Faculdade de Medicina, Universidade de São Paulo (FM/USP). Professor, Departamento de Medicina Preventiva, FM/USP. São Paulo/SP

- Brasil. E-mail: mscheffer@uol.com.br.

Artigo recebido em: 04/09/2012. Revisado em: 10/12/2012. Aprovado em: 24/12/2012. 
radiotherapy and surgeries. Diagnostic tests, medicines, orthosis and prosthesis were also denied. In $88 \%$ of the cases the judgment was favorable to the user, forcing the health plan company to provide the neglected coverage.

Keywords: Health Systems; Judicial Power; Private Medical Coverage.

\section{Introdução}

A judicialização da saúde é um fenômeno da atualidade que mobiliza pesquisadores, gestores, técnicos, membros do Poder Judiciário e do Ministério Público. O crescente número de cidadãos que buscam o direito à saúde nos tribunais gerou diversos estudos sobre ações judiciais que têm contribuído para a compreensão do funcionamento e das lacunas do sistema de saúde brasileiro.

Dentre as várias dimensões de análise da judicialização na saúde destacam-se aquelas que priorizam o comportamento do Judiciário, a conformidade com a legislação, as competências das instituições reclamadas nos tribunais e as características dos autores das ações. Mas também é possível, por meio das ações judiciais, traçar perfis das demandas e dos problemas de saúde, bem como analisar falhas e disfunções da regulação do sistema de saúde.(1)

Há evidências dos efeitos negativos das ações judiciais na gestão das políticas públicas e privadas de saúde, capazes de gerar iniquidades no acesso à saúde, expressar privilégios de determinados grupos e indivíduos em detrimento da coletividade, respondendo a lobbies e interesses particulares.(2),(3),(4) Mas é possível identificar também uma relação positiva entre acesso à Justiça e efetivação do direito, a partir do pressuposto de que a judicialização nem sempre é uma distorção a ser combatida, nem reside aí, necessariamente, um desvio do Judiciário em suas funções. ${ }^{(5),(6),(7)}$

\footnotetext{
1 PEPE, V.L.E.; VENTURA, M. (Org.). Manual indicadores de avaliação e monitoramento das demandas judiciais de medicamentos. Rio de Janeiro: Fundação Oswaldo Cruz, Escola Nacional de Saúde Pública Sergio Arouca, 2011. Disponível em: <http://www5.ensp.fiocruz.br/biblioteca/dados/ txt_975659982.pdf>. Acesso em: 27 ago.2012.

2 VIEIRA, F.S.; ZUCCHI, P. Distorções causadas pelas ações judiciais à política de medicamentos no Brasil. Revista Saúde Pública, São Paulo, v. 41, n. 2, p. 214-222, abr. 2007.

${ }^{3}$ MARQUES, S.B.; DALLARI, S.G. Garantia do direito social à assistência farmacêutica no Estado de São Paulo. Revista Saúde Pública, São Paulo, v. 41, n. 1, p. 101-107, fev. 2007.

${ }^{4}$ CHIEFFI, A.L.; BARATA, R.B. Judicialização da política pública de assistência farmacêutica e equidade. Cadernos de Saúde Pública, Rio de Janeiro, v. 25, n. 8, p. 1839-1349, ago. 2009.

${ }^{5}$ NOBRE, M.A.B. Da denominada "Judicialização da Saúde": pontos e contrapontos. In: NOBRE, M.A.B.; SILVA, R.A.D. (Coord.). O CNJ e os desafios da efetivação do Direito à Saúde. Belo Horizonte: Fórum, 2011. p. 353-366.

${ }^{6}$ SALAZAR, A.L.; GROU, K.B. A defesa da saúde em juízo: teoria e prática. São Paulo: Verbatim, 2009.

7 PEPE, V.L.E. et al. A judicialização da saúde e os novos desafios da gestão da assistência farmacêutica. Ciência \& Saúde Coletiva, Rio de Janeiro, vol. 15, n. 5, p. 2405-2414, ago. 2010.
} 
O estudo da judicialização da saúde tem abordado prioritariamente as ações que buscam obrigar o Sistema Único de Saúde (SUS) a fornecer medicamentos, insumos e procedimentos. ${ }^{(8)}$ É crescente, no entanto, o interesse em estudar o fenômeno das ações judiciais movidas contra planos e seguros de saúde. Nesse caso, destacam-se o estudo das coberturas assistenciais, ${ }^{(9),(10),(11)}$ o comportamento do Judiciário ${ }^{(12),}{ }^{(13)}$ e o desequilíbrio econômico causado pela interferência do Poder Judiciário nas relações contratuais entre clientes e planos de saúde.(14),(15)

A Constituição Federal de 1988 definiu a saúde como direito de todos e dever do Estado, estabeleceu o princípio da universalidade, atribuiu relevância pública ao setor e reconheceu a livre atuação da iniciativa privada. No entanto, somente dez anos depois, com a aprovação da Lei 9.656/98, ${ }^{(16)}$ os planos e seguros de saúde privados passaram a ser regulados pelo Estado.

A restrição de coberturas na assistência médica suplementar, tema recorrente nos tribunais, repercute na saúde e na vida da população usuária, bem como nos custos administrativos das empresas de planos e seguros de saúde, mas também interfere no sistema público de saúde, pois os limites de atendimento inscritos nos contratos de planos de saúde, previstos na legislação ou autorizados pela Agência Nacional de Saúde Suplementar (ANS), são absorvidos, em grande parte, pelo SUS.

A amplitude da cobertura depende da época da contratação do plano de saúde, pois há contratos "novos", assinados a partir de 1999; antigos, anteriores à Lei $n^{\circ}$ 9.656/98; e adaptados (por meio de um aditivo contratual) ou que sofreram migração (por meio da contratação de um novo plano de saúde na mesma operadora). Também depende do tipo de plano contratado (há segmentação

\footnotetext{
${ }^{8}$ ANDRADE, E.I.G. A judicialização da saúde e a Política Nacional de Assistência Farmacêutica no Brasil: gestão da clínica e medicalização da justiça. Revista Médica de Minas Gerais, Belo Horizonte, v. 18, n. 4 (Supl.4), p. S46-S50, 2008.

${ }^{9}$ OLIVEIRA, J.A.D. Demandas jurídicas por coberturas assistenciais: estudo de caso: CASSI. 2010. Dissertação (Mestrado em Saúde Pública) - Faculdade de Saúde Pública, Universidade de São Paulo, São Paulo.

${ }^{10}$ SCHEFFER, M. A exclusão de coberturas assistenciais nos planos de saúde privados. Saúde em Debate, Rio de Janeiro, v. 29, n. 71, p. 231-247, set./dez. 2005.

${ }^{11}$ PIRES, D.S.; SOUZA, J.M. Judicialização da saúde suplementar. Belo Horizonte: UNIMED-BH, 2008.

12 TRETTEL, D.B. Planos de saúde na visão do STJ e do STF. São Paulo: Verbatim, 2010.

${ }^{13}$ SOUZA, M.H.S. C. et al. A intervenção do Poder Judiciário no setor de saúde suplementar - tutelas antecipadas como instrumento de garantia da assistência à saúde no Brasil. Divulgação em Saúde para Debate, Rio de Janeiro, n. 37, p. 44-60, jan. 2007.

${ }_{14}$ GONÇALVES, T.; MACHADO, F. J. Judicialização da saúde suplementar. Sare - Sistema Anhanguera de Revistas Eletrônicas. 2011. Disponível em: <http://www.sare.unianhanguera.edu. br/index.php/ansem/article/view/4025>. Acesso em: 27 ago.2012.

${ }_{15}$ CUNHA, C.M. et al. A judicialização da saúde suplementar. uma análise econômica. 2011. Disponível em: <http://blog.newtonpaiva.br/seer_3/index.php/RevistaPos/article/viewFile/202/203>. Acesso em: 27 ago.2012

${ }^{16}$ BRASIL. Lei $n^{\circ}$ 9.656, de 3 de junho de 1998. "Dispõe sobre os planos e seguros privados de assistência à saúde". Disponível em: < http://www.planalto.gov.br/ccivil_03/Leis/L9656compilado. htm>. Acesso em: 29 mar. 2013.
} 
de coberturas ambulatoriais e hospitalares) e da forma de contratação (plano individual, familiar ou coletivo).

Os contratos antigos - que atingem cerca de 7,6 milhões de pessoas ${ }^{(17)}$ - ostentam cláusulas restritivas, assim como os contratos regidos pela Lei $n^{\circ}$ 9.656/98 trazem possibilidades de exclusões. Ou seja, há margens para flexibilidades que geram práticas abusivas e motivam queixas, reclamações e intensas disputas judiciais, sobretudo em torno das negações de coberturas.

Aproximadamente um quarto da população brasileira (47,9 milhões de pessoas) está vinculado a planos privados de assistência médica, segmento do mercado da saúde no qual operam 1.006 empresas que movimentaram uma receita em torno de $\mathrm{R} \$ 84,4$ bilhões em 2011. ${ }^{(18)}$

Assim, o estudo das ações judiciais relacionadas às restrições de coberturas busca trazer elementos para o aprimoramento da regulação e das práticas de um setor que tem grande impacto na conformação do financiamento e do funcionamento do sistema de saúde brasileiro.

\section{Materiais e métodos}

A pesquisa abrangeu o estado de São Paulo, unidade da federação que concentra a maior taxa de cobertura de saúde suplementar: Quase metade $(44,8 \%)$ da população do estado (6,8 milhões de usuários) está vinculada a planos de saúde. ${ }^{(19)}$

Foram selecionados e analisados os acórdãos de ações julgadas em segunda instância pelo Tribunal de Justiça do Estado de São Paulo (TJ-SP) de janeiro de 2009 a dezembro de 2010. Os acórdãos, redigidos, datados e assinados pelos juízes, contêm o resumo da ação e, após lavrados, suas conclusões são publicadas na internet, no site oficial do TJ-SP.

$\mathrm{Na}$ busca online, foram combinadas diversas palavras-chave: "plano de saúde"; "convênio médico", "convênio de saúde" e "seguro saúde". Em seguida, essas palavras-chave foram combinadas com as seguintes expressões: "cobertura"; "exclusão"; "negação"; "negativa"; "atendimento"; "atendimento médico"; "assistência"; e "assistência médica". No sentido de refinar a busca, outra combinação de palavras foi testada, dessa vez utilizando palavras genéricas que poderiam levar às ações judiciais que contêm exclusões de cobertura: "doença"; "doença preexistente"; "doença

17 AGÊNCIA NACIONAL DE SAÚDE SUPLEMENTAR. Caderno de Informação da Saúde Suplementar. Beneficiários, Operadoras e Planos. Rio de Janeiro: ANS, Junho 2012. Disponível em: <http://www.ans.gov.br/index.php/a-ans/sala-de-noticias-ans/numeros-do-setor/1624-novaedicao-do-caderno-de-informacao-da-saude-suplementar-junho-2012>. Acesso em: 27 ago.2012.

${ }^{18}$ Id. Ibid.

${ }^{19}$ Id. Ibid. 
infectocontagiosa/infecciosa"; "doença crônica"; "patologia"; "consulta"; "consulta médica"; "exame"; "cirurgia", "procedimento", "procedimento médico", "internação", "internação em UTI"; "atendimento hospitalar", "atendimento ambulatorial", "atendimento domiciliar".

Isso levou à identificação de 1.415 acórdãos. Após a leitura, excluídos os acórdãos referentes a outros assuntos demandados por usuários de planos de saúde, chegou-se ao quantitativo de 782 ações judiciais relacionadas à negação de coberturas pelos planos de saúde. Um questionário estruturado foi aplicado a todos os acórdãos selecionados, contendo perguntas sobre: número do acórdão no TJ-SP, data e resultado do julgamento em segunda instância, titularidade da ação judicial, demanda de cobertura citada no acórdão, situação de saúde, exclusão de órtese e prótese, alegação de doença preexistente, menção a caso de urgência e emergência, legislação aplicada e argumentação da decisão.

Foram definidos, como objetivos principais da pesquisa: descrever as principais situações e problemas de saúde levados aos tribunais em ações relacionadas à negação de atendimento, exclusão ou restrição de cobertura; analisar o comportamento do Judiciário nos julgamentos proferidos em segunda instância; apontar eventuais lacunas na regulamentação dos planos e seguros de saúde.

\section{Resultados e discussão}

O estudo analisou 782 decisões judiciais relacionadas à exclusão de cobertura de planos de saúde, julgadas em segunda instância pelo TJ-SP em 2009 e 2010.

A maioria dos julgados (88\%) foi favorável ao usuário, obrigando o plano de saúde a arcar com a cobertura negada (Tabela 1).

Em 7,5\% das decisões, o juiz acatou o argumento do plano de saúde e negou a cobertura; em $4 \%$ dos casos concedeu parte da cobertura solicitada. O Código de Defesa do Consumidor (CDC) é a legislação mais citada nas argumentações judiciais favoráveis à cobertura; ele aparece em $59 \%$ das decisões analisadas.

Tabela 1. Ações judiciais no TJ-SP, relacionadas à cobertura de planos de saúde, segundo decisão de segunda instância, 2009 e 2010, São Paulo.

\begin{tabular}{lcc}
\hline Resultados & $\mathrm{N}^{\circ}$ de decisões & $\%$ \\
\hline Favorável à cobertura & 690 & 88,24 \\
Contrário à cobertura & 59 & 7,54 \\
Parcialmente favorável & 32 & 4,09 \\
Outras decisões & 1 & 0,13 \\
\hline Total & 782 & 100,00 \\
\hline
\end{tabular}


Em seguida vêm a Lei n 9.656/98, com 15\% de citações; o Código Civil (13\%) e a Constituição Federal ( 6,5\%), dentre outras legislações mencionadas.

Dentre as doenças ou grupos de doenças mais citados nas ações analisadas (Tabela 2), estão os diversos tipos de câncer (leucemia, linfomas, mama, próstata, colo de útero etc.), em 218 ações judiciais (36,57\%). Em segundo lugar estão as doenças do aparelho circulatório, incluindo Acidente Vascular Cerebral (AVC), infarto e outros problemas cardíacos, com 116 ações judiciais (19,46\%).

Tabela 2. Ações judiciais no TJ-SP, relacionadas à cobertura de planos de saúde, segundo grupos de doenças excluídas citadas nos acórdãos, 2009 e 2010,

São Paulo.

\begin{tabular}{lcc}
\hline Grupos de doenças mencionados & $\begin{array}{c}\text { Citações nas } \\
\text { ações judiciais }\end{array}$ & $\%$ \\
\hline Neoplasmas (Tumores) & 218 & 36,57 \\
Doenças do aparelho circulatório & 116 & 19,46 \\
Doenças do sistema osteomuscular e do tecido conjuntivo & 58 & 9,73 \\
Obesidade mórbida & 42 & 7,04 \\
Causas externas/acidentes & 24 & 4,02 \\
Insuficiência renal & 18 & 3,02 \\
Doenças infecciosas/AIDS/hepatite, meningite & 17 & 2,85 \\
Doenças do olho & 17 & 2,85 \\
Doenças congênitas/ degenerativas/síndromes & 11 & 1,84 \\
Transtornos mentais/dependência química & 11 & 1,84 \\
Diabetes & 10 & 1,67 \\
Doenças do fígado & 7 & 1,17 \\
Outras doenças & 47 & 7,88 \\
\hline Total & 596 & 100,00 \\
\hline
\end{tabular}

Obs.: O acórdão analisado pode indicar mais de uma doença/problema de saúde. Nem todos os acórdãos mencionam o diagnóstico do paciente.

Em seguida vêm as doenças do sistema osteomuscular, artroses, hérnia de disco, problemas ortopédicos e de coluna; os casos de obesidade mórbida; as causas externas, principalmente acidentes e traumatismos; a insuficiência renal, doenças infecciosas, dentre outros agravos.

Quimioterapia e radioterapia para tratamento do câncer, o que inclui quimioterápicos para uso ambulatorial e domiciliar, são os procedimentos mais negados: correspondem a 35,95\% das ações judiciais que mencionam procedimentos médicos excluídos pelos planos de saúde. Em seguida vêm as cirurgias (mastectomia, cirurgias cardíacas e bariátricas, aneurisma, vesícula etc.); sessões de fisioterapia, fonoaudiologia e reabilitações; hemodiálise; transplantes, dentre outros procedimentos (Tabela 3). 
Tabela 3. Ações judiciais no TJ-SP, relacionadas à cobertura de planos de saúde, segundo tipos de procedimentos médicos excluídos, 2009 e 2010, São Paulo.

\begin{tabular}{lcc}
\hline Tipo de procedimento excluído & $\begin{array}{c}\text { Citações nas } \\
\text { ações judiciais }\end{array}$ & $\%$ \\
\hline Quimioterapia/Radioterapia & 133 & 35,95 \\
Cirurgia & 124 & 33,51 \\
Fisioterapia/Fonoaudiologia/Reabilitações & 24 & 6,49 \\
Diálise/Hemodiálise & 21 & 5,68 \\
Transplantes & 18 & 4,86 \\
Oxigenoterapia & 13 & 3,51 \\
Parto & 6 & 1,62 \\
Outros procedimentos & 31 & 8,38 \\
\hline Total & 370 & 100,00 \\
\hline
\end{tabular}

Obs.: O acórdão analisado pode indicar mais de um procedimento médico excluído. Nem todos os acórdãos mencionam a exclusão de procedimento.

Nas 18 decisões analisadas que mencionaram a negativa de transplantes, os mais excluídos são os de medula (nove ações judiciais), fígado (cinco ações) e pâncreas (três ações), além de um caso de transplante de rim. Em todos os casos os planos de saúde foram obrigados a arcar com os custos. A regulamentação em vigor determina a obrigatoriedade de transplantes de rins, córneas, autólogos e heterólogos de medula óssea.

Quanto aos diversos insumos negados pelos planos de saúde, segundo as decisões judiciais analisadas (Tabela 4), prevalecem as órteses e próteses, principalmente stents, marcapassos e próteses ortopédicas (de quadril, joelho etc.), com 243 menções (40,7\%). Os medicamentos não cobertos (citados em 29,4\% das ações judiciais) são de alto custo e uso hospitalar, mas também alguns fármacos para tratamentos ambulatoriais, em hospital-dia e em domicílio. Dentre os exames negados, em $21,9 \%$ dos acórdãos, os mais citados são o PET Scan ou PET/CT (exame por imagem cada vez mais usado na oncologia, neurologia e cardiologia), mas também tomografia, ressonância magnética, ultrassonografia e cateterismo.

Foram mencionadas em várias ações judiciais, sem especificação do problema de saúde do paciente, negativas de autorização de internação (80 casos), de internação em UTI (19 casos) e de atendimento domiciliar ou home care (18 casos), além da não cobertura de remoções e transporte de pacientes, de despesas hospitalares e honorários médicos.

Quanto aos argumentos usados pelas operadoras em juízo, para justificar a negação da cobertura, destacam-se: o procedimento não consta no Rol de 
Tabela 4. Ações judiciais no TJ-SP, relacionadas à cobertura de planos de saúde, segundo insumos excluídos, 2009 e 2010, São Paulo.

\begin{tabular}{lcc}
\hline Tipo de insumo & $\begin{array}{c}\text { Citações nas } \\
\text { ações judiciais }\end{array}$ & $\%$ \\
\hline Órtese e prótese & 243 & 40,71 \\
Medicamentos & 176 & 29,48 \\
Exame diagnóstico & 131 & 21,94 \\
Materiais cirúrgicos & 27 & 4,52 \\
Outros insumos & 20 & 3,35 \\
\hline Total & 597 & 100,00 \\
\hline
\end{tabular}

Obs.: O acórdão analisado pode indicar mais de um insumo excluído. Nem todos os acórdãos mencionam a exclusão de insumos.

Procedimentos da ANS; o contrato possui cláusula que exclui a cobertura solicitada (situação comum nos planos antigos, assinados antes da Lei ${ }^{\circ} 9.656 / 98$ ); ou a doença é preexistente, que "é aquela que o consumidor ou seu responsável saiba ser portador ou sofredor à época da contratação do plano".(20)

Outras alegações foram o não cumprimento do prazo de carência (dois anos para doenças preexistentes, 300 dias para partos etc.); o fato de o hospital ou serviço utilizado não ser credenciado do plano de saúde; o tratamento médico estar fora de diretriz clínica segundo auditoria do plano de saúde; e a não obrigatoriedade de o plano de saúde cobrir medicamentos, sobretudo de uso ambulatorial, domiciliar e oral.

A finalidade estética do procedimento foi a justificativa para a não cobertura de diversos casos de reconstrução de mama devido a câncer e em casos de redução de estômago devido a obesidade mórbida. E ainda a alegação de que, no caso de planos de saúde coletivos (pessoa jurídica, associação etc.), prevalece o contrato entre as partes, não há relação de consumo e não se aplicaria o CDC nem a legislação vigente para os planos individuais. Por fim, foi alegado por algumas operadoras que a garantia irrestrita à saúde é dever do Estado e não dos planos de saúde.

Nos acórdãos analisados foram movidas ações contra 140 empresas de planos de saúde; 66 delas aparecem em uma ação judicial e 27 empresas são reclamadas em duas ou mais ações.

As exclusões reclamadas na Justiça referem-se principalmente a tratamentos e procedimentos de alto custo e alta complexidade, o que dá a dimensão do potencial de aportes públicos ao sistema supletivo, ainda que não seja possível

\footnotetext{
${ }^{20}$ BRASIL. Resolução CONSU, n 2, de 4 de novembro de 1998. "Dispõe sobre a definição de cobertura às doenças e lesões preexistentes previstas no inciso XII do art. 35 e no art. 11 da lei no 9656/98". Diário Oficial da União, Brasília (DF), n. 211, 1998.
} 
a quantificação dos recursos despendidos pelo SUS para atender aquilo que não é coberto pelos planos de saúde.

Vale ressaltar que os dois grupos de doenças mais citados nos acórdãos - câncer e doenças cardiovasculares - referem-se às principais causas de mortalidade e morbidade no estado de São Paulo, o que indica que a judicialização, nesses casos, pode ser indício de um problema de grande magnitude, pois diz respeito a problemas de saúde frequentes na população.

É possível supor que a dimensão da exclusão de coberturas é maior do que os casos que chegam à justiça. Como são situações urgentes, de vida e saúde, diante da negativa de cobertura os cidadãos e famílias tendem a arcar com os custos particulares ou mesmo buscam atendimento na rede pública, o que sobrecarrega e onera o sistema público de saúde.

O estudo mostra, 13 anos depois do início da vigência da Lei $n^{\circ} 9.656 / 98$, que ainda existem falhas da legislação e dificuldades de a ANS exercer em plenitude seu papel regulador e fiscalizador do setor.

A diversidade de justificativas das empresas de planos de saúde para a restrição da assistência indica a complexidade da regulação, mas também aponta para possibilidades concretas de aperfeiçoamento da legislação. Assegurado o direito adquirido, incentivar a transição dos contratos antigos para as regras atuais, a atualização do Rol de Procedimentos da ANS em menor lapso temporal, com ampliação do leque de coberturas obrigatórias, assim como a implementação de diretrizes clínicas e consensos terapêuticos, são medidas que poderiam evitar inúmeras ações judiciais.

As decisões analisadas, com $88 \%$ de ganho de causa a favor do usuário, apontam no sentido de que não há fundamento jurídico na maioria das exclusões de cobertura, tanto naquelas expressas nos contratos antigos quanto naquelas que encontram respaldo na atual legislação.

Antes de recorrer aos tribunais, muitos usuários por certo tentaram a solução da negação de cobertura por meio administrativo, junto ao plano de saúde, ou mesmo podem ter levado a queixa à ANS e a órgãos de defesa do consumidor. Cabe aqui discutir a capacidade limitada dessas instâncias em acolher adequadamente e intermediar conflitos entre clientes e operadoras de planos de saúde, visando à solução do problema sem a necessidade da ação judicial.

\section{Considerações finais}

O desempenho da economia tem alavancado os planos de saúde, que são cada vez mais ofertados pelos empregadores; são prioridade na pauta dos sindicatos e objeto de desejo dos indivíduos e famílias que perderam a confiança no sistema público de saúde. 
Há, nos grandes centros, uma proliferação de planos baratos e populares, com rede credenciada de médicos, hospitais e laboratórios, limitada e insuficiente, com dificuldade de prestar assistência adequada, o que poderá levar a mais restrições de atendimentos.

Como consequência da ampliação da negação de coberturas, usuários de planos de saúde baterão às portas da Justiça, sobrecarregando os tribunais e gerando novos custos administrativos ao sistema de saúde suplementar.

Cresce a chance de parte do mercado de assistência médica suplementar buscar sua sobrevivência às custas das restrições praticadas e do consequente subsídio indireto do Sistema Único de Saúde, que arca com as despesas dos atendimentos negados pelos planos de saúde.

Previsto na Lei $n^{\circ} 9.656 / 98$, o ressarcimento ao SUS ainda não se mostrou inteiramente viável na prática, sendo irrisório o montante de recursos repassados ao erário quando clientes de planos de saúde são atendidos pela rede pública. $A$ solução do problema requer o aprimoramento da regulamentação específica, a atuação firme da agência reguladora, a expansão dos planos de saúde compatível com a capacidade de atendimento da rede credenciada e a revisão da relação entre o público e o privado na saúde no Brasil, o que exigirá a transformação dos modelos assistenciais, do financiamento e da prestação de serviços.

\section{Referências}

AGÊNCIA NACIONAL DE SAÚDE SUPLEMENTAR. Caderno de Informação da Saúde Suplementar: Beneficiários, Operadoras e Planos. Rio de Janeiro: ANS, Junho 2012. Disponível em: <http://www.ans.gov.br/index.php/a-ans/sala-denoticias-ans/numeros-do-setor/1624-nova-edicao-do-caderno-de-informacaoda-saude-suplementar-junho-2012>. Acesso em: 27 ago. 2012.

ANDRADE, E.I.G. A judicialização da saúde e a Política Nacional de Assistência Farmacêutica no Brasil: gestão da clínica e medicalização da justiça. Revista Médica de Minas Gerais, Belo Horizonte, v. 18, n. 4 (Supl.4), p. S46-S50, 2008.

CHIEFFI, A.L.; BARATA, R.B. Judicialização da política pública de assistência farmacêutica e equidade. Cadernos de Saúde Pública, Rio de Janeiro, v. 25, n. 8, p. 1839-1349, ago. 2009.

CUNHA, C.M. et al. A judicialização da saúde suplementar: uma análise econômica. 2011. Disponível em: <http://blog.newtonpaiva.br/seer_3/index.php/ RevistaPos/article/viewFile/202/203>. Acesso em: 27 ago. 2012. 
GONÇALVES, T.; MACHADO, F.J. Judicialização da saúde suplementar. Sare - Sistema Anhanguera de Revistas Eletrônicas. 2011. Disponível em: <http:// www.sare.unianhanguera.edu.br/index.php/ansem/article/view/4025>. Acesso em: 27 ago. 2012.

MARQUES, S.B.; DALLARI, S.G. Garantia do direito social à assistência farmacêutica no Estado de São Paulo. Revista Saúde Pública, São Paulo, v. 41, n. 1, p. 101-107, fev. 2007.

NOBRE, M.A.B. Da denominada "Judicialização da Saúde": pontos e contrapontos. In: NOBRE, M.A.B.; SILVA, R.A.D. (Coord.). O CNJ e os desafios da efetivação do Direito à Saúde. Belo Horizonte: Fórum, 2011. p. 353-366.

OLIVEIRA, J.A.D. Demandas jurídicas por coberturas assistenciais: estudo de caso: CASSI. 2010. Dissertação (Mestrado em Saúde Pública) - Faculdade de Saúde Pública, Universidade de São Paulo, São Paulo.

PEPE, V.L.E. et al. A judicialização da saúde e os novos desafios da gestão da assistência farmacêutica. Ciência \& Saúde Coletiva, Rio de Janeiro, vol. 15, n. 5, p. 2405-2414, ago. 2010.

; VENTURA, M. (Orgs.). Manual indicadores de avaliação e monitoramento das demandas judiciais de medicamentos. Rio de Janeiro: Fundação Oswaldo Cruz, Escola Nacional de Saúde Pública Sergio Arouca, 2011. Disponível em: $<$ http://www5.ensp.fiocruz.br/biblioteca/dados/txt_975659982.pdf>. Acesso em: 27 ago. 2012.

PIRES, D.S.; SOUZA, J.M. Judicialização da saúde suplementar. Belo Horizonte: UNIMED-BH, 2008.

SALAZAR, A.L.; GROU, K.B. A defesa da saúde em juízo: teoria e prática. São Paulo: Verbatim, 2009.

SCHEFFER, M. A exclusão de coberturas assistenciais nos planos de saúde privados. Saúde em Debate, Rio de Janeiro, v. 29, n. 71, p. 231-247, set./dez. 2005.

SOUZA, M.H.S.C. et al. A intervenção do Poder Judiciário no setor de saúde suplementar - tutelas antecipadas como instrumento de garantia da assistência à saúde no Brasil. Divulgação em Saúde para Debate, Rio de Janeiro, n. 37, p. 44-60, jan. 2007.

TRETTEL, D.B. Planos de saúde na visão do STJ e do STF. São Paulo: Verbatim, 2010.

VIEIRA, F.S.; ZUCCHI, P. Distorções causadas pelas ações judiciais à política de medicamentos no Brasil. Revista Saúde Pública, São Paulo, v. 41, n. 2, p. 214-222, abr. 2007. 